\title{
Inflammatory cytokines gene expression in bone tissue from patients with chronic rhinosinusitis - a preliminary study*
}

\author{
A. Tuszynska ${ }^{1}$, A. Krzeski $^{1}$, M. Postuła ${ }^{2}$, L. Paczek $^{3}$, A. Wyczalkowska-Tomasik $^{3}$, \\ B. Górnicka ${ }^{4}$, R. Pykalo ${ }^{4}$ \\ 1 Department of Otolaryngology, Medical University of Warsaw, Warsaw, Poland \\ 2 Department of Experimental and Clinical Pharmacology, Medical University of Warsaw, Warsaw, Poland \\ 3 Department of Immunology, Transplantology and Internal Diseases, Medical University of Warsaw, Warsaw, \\ Poland \\ 4 Department of Pathology, Medical University of Warsaw, Warsaw, Poland
}

\begin{abstract}
SUMMARY
Background: It is unclear whether mucosal inflammation has an effect on the bone underneath the mucosa in patients with chronic rhinosinusitis (CRS).

Objectives: The aim of this study was to evaluate inflammatory cytokine gene expression in bone tissue taken from patients who had undergone endoscopic sinus surgery (ESS) for CRS. Methods: A group of 49 consecutive patients, diagnosed with CRS based on EPOS 2007 criteria, was enrolled in the study. Based on the histopathological findings of the mucosal and bone tissues, we evaluated the extent of inflammation. Expression of target genes - interleukin (IL)$1 \beta, I L-6, I L-11$, tumor growth factor (TGF)- $\beta$ and tumor necrosis factor (TNF)- $\alpha$ - in samples of the ethmoid bone taken during endoscopic sinus surgery for CRS was analysed by real-time PCR.

Results: Based on the histopathological findings in the studied specimens, we found signs of osteitis in 5 patients. In the studied population, we found significant differences between patients with and without osteitis, with respect to IL-6, IL-11 and TNF- $\alpha$ gene expression in bone tissue.
\end{abstract}

Conclusion: We have demonstrated that in some patients with CRS and coexisting signs of osteitis, gene expression of some inflammatory markers is increased.

Key words: chronic rhinosinusitis, bone tissue, osteitis, real-time PCR, gene expression, interleukin

\section{INTRODUCTION}

Rhinosinusitis is a multifactorial inflammatory process involving the mucosa of the nose and one or more sinuses. Chronic rhinosinusitis (CRS) is defined as inflammation of the nose and the paranasal sinuses characterized by two or more symptoms of over 12 weeks' duration, one of which should be either nasal blockage/obstruction/congestion or nasal discharge (anterior/posterior nasal drip), \pm facial pain/pressure, \pm reduction or loss of smell; and either endoscopic signs of polyps and/or mucopurulent discharge primarily from the middle meatus and/or oedema/mucosal obstruction primarily in the middle meatus, and/or CT changes showing mucosal changes within the ostiomeatal complex and/or sinuses ${ }^{(1)}$. CRS may take a clinical form without the development of nasal polyps or with nasal polyps. According to most recent studies, these are two distinct categories of the disease, the development of which involves the participation of different inflammatory cells as well as a different progress of histopathological changes occurring in the mucosa. Factors contributing can be mucociliairy impairment, bacterial infection, allergy, swelling of the mucosa for another reason, or rarely physical obstructions caused by morphological and anatomical variations in the nasal cavity or paranasal sinuses ${ }^{(1)}$.

Although the main inflammatory site of rhinosinusitis is the nasal mucosa, it is often unclear whether mucosal inflammation has an effect on the bone under the mucosa and whether it causes osteitis or bone remodeling. Bone changes have been observed clinically, radiographically and histologically in CRS for many years. Such changes have traditionally been considered reactive and therefore not of significant clinical relevance. However, more recent work suggests that the bone may actual- 
ly play an active part in the disease process and that, at a minimum, the inflammation associated with CRS may spread through the Haversian system within the bone. Recently, histopathological and radiological aspects of bone remodeling and osteitis in CRS have been studied, and it is known that many histological similarities in bone involvement exist between CRS and osteomyelitis of long bones ${ }^{(2-6)}$. Osteitis is defined as a superficial inflammatory process in bone or bone without a marrow, but the terms osteomyelitis and osteitis are frequently used interchangeably ${ }^{(7)}$. Osteitis or bone remodeling in CRS usually results in a mixture of osteosclerosis and osteolytic lesions, but it is evident that the homeostatic balance between osteoclasts and osteoblasts is disrupted, regardless of the final phenotypes. The pressure effects of polyps and the presence of inflammatory mediators such as IL-1, tumor necrosis factor (TNF), and prostaglandin have been proposed as causes of bone remodeling and osteitis ${ }^{(8,9)}$. In specimens of the mucosa from paranasal sinuses obtained from patients with CRS, the concentration of IL-1, IL-6, IL-8, TNF- $\alpha$, IL-3, GMCSF, ICAM-1, VCAM-1, selectins, myeloperoxydase and ECP was found to be higher in comparison to specimens from the controls without CRS ${ }^{(10-14)}$. Thus, the aim of this study was evaluation of inflammatory cytokines genes expression in bone tissue taken from the patients who had undergone endoscopic sinus surgery for CRS with or without polyposis.

\section{MATERIALS AND METHODS \\ Patients}

All the study patients were informed of the study and gave written consent before study procedures were started. The local ethics committee of the Medical University of Warsaw approved the study protocol and the informed consent form. This study was conducted in accordance with the latest version of Helsinki Declaration.

A total group of a consecutive 49 patients with diagnosis of CRS based on EPOS 2007 criteria undergoing endoscopic sinus surgery for CRS were enrolled in the study. CRS was confirmed by symptoms, endoscopy, and computed tomography (CT) (Lund-Mackay score range 8-16). At the preoperative visit, all patients were questioned about the time of nasal polyps (NP) evolution, the presence of other chronic diseases, smoking habit, family history of NP, and history of allergic rhinitis and/or allergy. Bilateral nasal polyps were diagnosed based on history, clinical examination, nasal endoscopy, and sinus CT scan. Nasal polyposis was defined as presence of endoscopically visible bilateral polyps growing from the middle nasal meatus into the nasal cavities and affecting bilaterally more than 1 paranasal sinus according to the CT scan. Nasal polyps were identified in all patients by the following criteria: nasal symptoms (obstruction, anosmia, sneezing, rhinorrhea, itching) and visualization of polyps by nasal endoscopy. Each nasal symptom was scored from 0 to 3 ( $0=$ no symptom; $1=$ mild symptom [just noticeable]; 2 = moderate symptom [annoying]; $3=$ severe symptom [distress]) so that maximal nasal score was 15 of 15 .

\section{Surgery}

All patients were asked to stop any medication 1 month before the surgery. Procedures were performed by the same surgeon based on the extension of patient's disease. After obtaining informed consent, samples of the ethmoid bone was taken from all the patients who had undergone endoscopic sinus surgery for CRS with or without polyposis.

\section{Histology}

The tissues were fixed in 10\% formalin and decalcified in EDTA. The tissue blocks were sectioned in $5 \mu \mathrm{m}$ sections and stained with haematoxylin and eosin (H-E) for routine light microscopy. Based on histopathological findings, the mucosal grades were determined by the quantities of infiltrated inflammatory cells (lymphocytes, eosinophils, monocytes) on a scale of 0-3 as follows: 0 : lack of inflammatory cells; 1 : 1-25 inflammatory cells; $2: 26$ - 50 inflammatory cells; $3:>50$ inflammatory cells. The same pathologist who was blinded to the patient's history and CT findings reviewed the specimens and graded

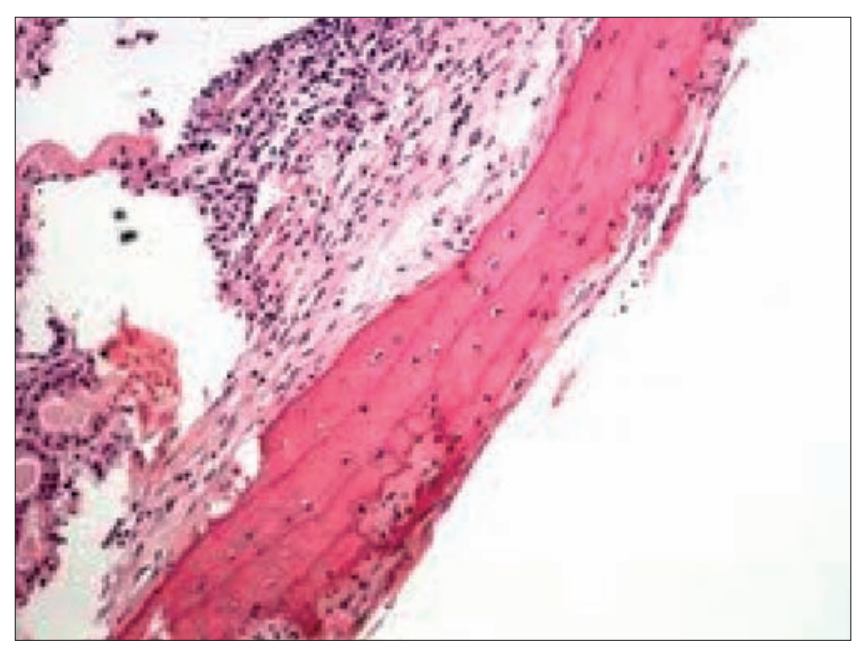

Figure 1. Bony trabecula of the ethmoid without inflammatory infiltration (10 x magnification).

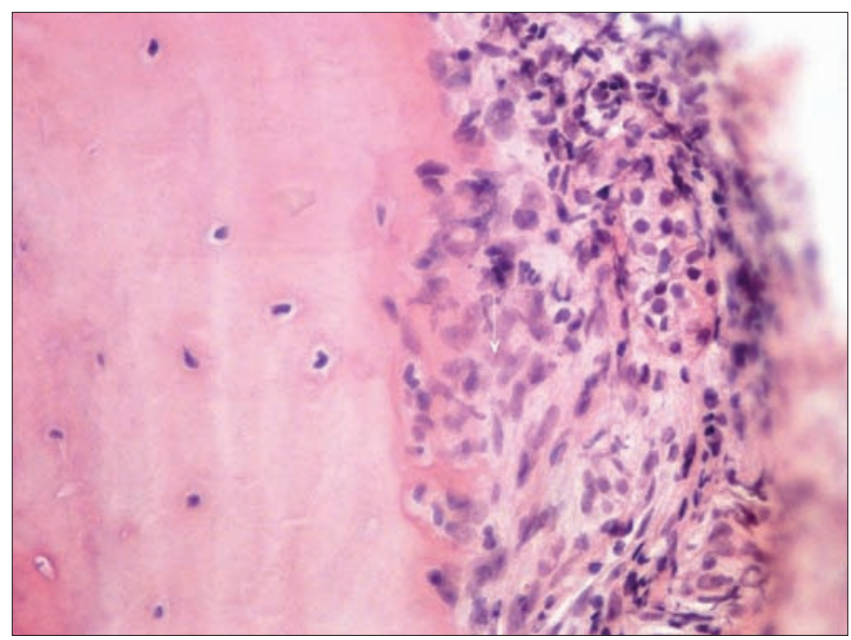

Figure 2. Inflammatory infiltration (lymphocytes, hystiocytes) in the bony trabecula of the ethmoid bone ( $40 \mathrm{x}$ magnification). 
both the mucosal and bony changes. The pathological criteria for osteitis were the presence of bony remodeling, with new immature woven bone formation seen overlying organized lamellar bone (samples of bone tissue without osteitis and bone tissue with osteitis are given in Figures 1 and 2).

\section{$R T-P C R$}

Samples of bone tissue were dissected from the overlying mucosa during surgery, each time under the same circumstances - in the sterile conditions of the operating room, prepared immediately after removal, by the same person using the same instruments and a microscope for guidance. The obtained tissue was rapidly frozen in special DNA'se- and RNA'se- free test tubes and stored at $-80^{\circ} \mathrm{C}$. The frozen samples were homogenized and then, total RNA was extracted with the usage of RNeasy Mini Kit (Qiagen) according to a modified method. The concentration of isolated RNA was determined by optical density at $\lambda=260 \mathrm{~nm}$, using Spectrophotometer UV/Vis ND-1000 (NanoDrop). RNA integrity was assessed by electrophoresis through $1.5 \%$ agarose gel after staining with ethidium bromide (Sigma) $2 \mu \mathrm{g}$ of total RNA was transcribed by SuperScript III First-Strand Synthesis for RT-PCR (Invitrogen). Expression of target genes: IL-1 $\beta$, IL-6, IL-11, TGF- $\beta$ and TNF- $\alpha$ was analysed by real-time PCR using the ABI PRISM 7700 Sequence Detector system. Primers and probes (Assays-on-Demand) for all genes were purchased from Applied Biosystems. Glyceraldehyde-3phosphate dehydrogenase (GAPDH) (Applied Biosystems) served as the endogenous control. Relative quantity of investigated gene expression was calculated using the comparative method described in the User Bulletin No 2 provided by Applied Biosystems. Expression of target genes were given as the threshold cycle $(\mathrm{Ct})$. The Threshold Cycle $(\mathrm{Ct})$ reflects the cycle number at which the fluorescence generated within a reaction crosses the threshold. Cycle number (in qPCR) at which the fluorescence generated within a reaction well exceeds the defined threshold. The threshold was arbitrarily defined by the manufacturer to reflect the point during the reaction at which a sufficient number of amplicons have accumulated. $\Delta \mathrm{Ct}$ shows the difference between the threshold cycle of a sample assay and the threshold cycle of the corresponding endogenous reference: $\Delta \mathrm{Ct}=\mathrm{Ct}(\operatorname{target})-\mathrm{Ct}($ endogenous control).

\section{Statistics}

Statistical data was calculated using STATISTICA version 6.0 program including t-test, Cochran test, one-way analysis of variance, Fisher exact test and LSD-test of planned comparison.

\section{RESULTS}

Patient age ranged from 16-79 years, with a mean age of 39.7 years and more than $54 \%$ of patients were younger than 30 years old. Twenty-one patients (42.85\%) were males and 28
Table 1. Clinical characteristics of the studied population.

\begin{tabular}{|c|c|}
\hline \multicolumn{2}{|l|}{ Clinical characteristics } \\
\hline Men & 22 \\
\hline Age & Mean 39,7 (Range 16,1- 79,2) \\
\hline Rhinosinusitis & $21(42 \%)$ \\
\hline Rhinosinusitis + polyps & $29(58 \%)$ \\
\hline $\begin{array}{l}\text { Duration of symptoms } \\
\text { (years) }\end{array}$ & Mean 7 (range 1-25) \\
\hline$<1$ year & $5(10 \%)$ \\
\hline $1-3$ years & $10(20 \%)$ \\
\hline 3-5 years & $10(20 \%)$ \\
\hline$>5$ years & $23(46 \%)$ \\
\hline \multicolumn{2}{|c|}{$\begin{array}{l}\text { Staging of CRS } \\
\text { (Lund and Mackay system) }\end{array}$} \\
\hline $0-5$ & $10(20 \%)$ \\
\hline 6-11 & $14(28 \%)$ \\
\hline $12-17$ & $17(34 \%)$ \\
\hline $18-24 z Z$ & $9(18 \%)$ \\
\hline
\end{tabular}

CRS- chronic rhinosinusitis

Table 2. Symptoms of chronic rhinosinusitis in the studied population based on the EP3OS 2007 criteria.

\begin{tabular}{lcc}
\hline Symptoms & Number of Patients & Percentage \\
\hline Group A & 35 & $70 \%$ \\
Group B & 7 & $14 \%$ \\
Group C & 7 & $14 \%$ \\
Group D & 1 & $2 \%$ \\
\hline
\end{tabular}

Group A- nasal blockage/obstruction/congestion+nasal discharge (anterior/posterior nasal drip)+facial pain/pressure+reduction or loss of smell; Group B- nasal blockage/obstruction/congestion+nasal discharge (anterior/posterior nasal drip)+facial pain/pressure; Group C- nasal blockage/obstruction/congestion+nasal discharge (anterior/ posterior nasal drip)+reduction or loss of smell; Group D- nasal blockage/obstruction/congestion+nasal discharge (anterior/ posterior nasal drip).

(57.14\%) were females. In this studied population, duration of symptoms of CRS were from 1 to 25 years (mean 7 years). Clinical characteristics of this population are shown in Tables 1 and 2.

Histological examination of mucosal tissues stained with haematoxylin and eosin revealed an intense eosinophilic infiltrate in $16(32 \%)$ of the 50 patients with CRS studied. No important tissue eosinophilia was visualized in $11(22 \%)$ of these patients, both of them belonging to the non-allergic group (Table 3). Gene expression levels and the calculated normalized $\mathrm{Ct}(\Delta \mathrm{Ct})$ value for the associated samples in whole studied population are given in Table 4 and 5 . There was no correlation between $\Delta \mathrm{Ct}$ of IL-1 $\beta$, IL-6, IL-11 and sex, Lund and Mackay score, or mean duration of CRS symptoms. The $\Delta \mathrm{Ct}$ values of IL-1 $\beta$ were increased only in patients $(\mathrm{n}=6)$ with eosinophilic infiltration $>50$ in comparison to less severe inflammatory cells infiltration $(\Delta \mathrm{Ct}$ : $71.56 \pm 11.79$ vs $46.64 \pm$ 
Table 3. Inflammatory cells upon histological examination of the mucosal tissue samples.

\begin{tabular}{lccc}
\hline Number of cells & Eosinophils & Neutrophils & Monocytes \\
\hline Group & & & \\
0 & 11 pts $(22 \%)$ & 41 pts $(82 \%)$ & 1 pts $(2 \%)$ \\
1 & 23 pts $(46 \%)$ & 8 pts $(16 \%)$ & 40 pts $(80 \%)$ \\
2 & 10 pts $(20 \%)$ & 1 pts $(2 \%)$ & 9 pts $(18 \%)$ \\
3 & 6 pts $(12 \%)$ & 0 pts $(0 \%)$ & 0 pts $(0 \%)$ \\
\hline
\end{tabular}

0: Lack of cells; $1: 1$ - 25 cells; $2: 26$ - 50 cells; $3:>50$ cells

Table 4. Calculated normalized $\mathrm{Ct}(\Delta \mathrm{Ct})$ values of expressed genes in the studied populations.

\begin{tabular}{lccc}
\hline & Mean & SD & Range \\
\hline$\Delta$ Ct IL-1 $\beta$ gene & 11.50 & 2.39 & $4.26-16.06$ \\
$\Delta$ Ct IL-6 gene & 14.31 & 2.84 & $7.30-20.51$ \\
$\Delta$ Ct IL-11 gene & 14.32 & 2.46 & $8.91-19.81$ \\
$\Delta$ Ct TNF $\alpha$ gene & 11.49 & 2.10 & $5.15-15.16$ \\
$\Delta$ Ct TGF $\beta$ gene & 1.33 & 1.65 & $-1.33-5.04$ \\
\hline
\end{tabular}

9.51; $\mathrm{p} \leq 0.027$, respectively), and $\Delta \mathrm{Ct}$ values of IL-6 were increased in patients $(\mathrm{n}=15)$ with eosinophilic infiltration $>25$ $(\Delta \mathrm{Ct}: 14.89 \pm 2.64$ vs $13.01 \pm 2.92 ; \mathrm{p} \leq 0.03$, respectively) and in patients $(\mathrm{n}=9)$ with neutrophilic infiltration $(\Delta \mathrm{Ct}$ : $14.72 \pm 2.67$ vs $12.51 \pm 3.02 ; \mathrm{p} \leq 0.03$, respectively). There was no such correlation with respect to $\Delta \mathrm{Ct}$ values of IL-11, TNF- $\alpha$ and TGF$\beta$. We also found increased $\Delta \mathrm{Ct}$ values of $\mathrm{TNF}-\alpha$ in patients with CRS and nasal polyps in comparison to patients with CRS without nasal polyps $(\Delta \mathrm{Ct}$ : $10.77 \pm 1.80$ vs $12.02 \pm 2.17$; $\mathrm{p} \leq$ 0.04 , respectively). There was no such correlation with respect to $\Delta \mathrm{Ct}$ values of IL-11, TGF- $\beta$, IL-6, IL-1 $\beta$.

Based on the histopathological findings in the studied samples, we found signs of osteitis in 5 patients out of the studied population (3 men among them) (Figure 2). There was no significant difference between the group without osteitis (group A) (Figure 1) and with osteitis (group B) with respect to age, duration of CRS, nasal polyps occurrence based on endoscopic

Table 5. Comparison of the clinical characteristics of groups of patients with and without symptoms/signs of osteitis.

\begin{tabular}{lccc}
\hline & Group A & Group B & p-value \\
\hline No. of patients & 44 & 5 & $\leq 0.643$ \\
Age & $38.64 \pm 15.44$ & $46.4 \pm 20.27$ & $\leq 0.32$ \\
Duration of CRS & $6.73 \pm 5.49$ & $9.75 \pm 3.86$ & $\leq 0.289$ \\
Nasal polyps & $27 \mathrm{pts}$ & $2 \mathrm{pts}$ & $\leq 0.638$ \\
Asthma & $10 \mathrm{pts}$ & $2 \mathrm{pts}$ & $\leq 0.5819$ \\
NSAID's sensitivity & $5 \mathrm{pts}$ & $2 \mathrm{pts}$ & $\leq 0.138$ \\
\hline IL-1 $\beta$ gene & $11.72 \pm 2.09$ & $9.06 \pm 4.33$ & 0.31 \\
IL-6 gene & $14.37 \pm 2.44$ & $9.67 \pm 3.18$ & 0.0003 \\
IL-11 gene & $14.56 \pm 2.40$ & $11.66 \pm 1.59$ & 0.02 \\
TNF $\alpha$ gene & $11.47 \pm 1.88$ & $8.64 \pm 2.57$ & 0.0035 \\
TGF $\beta$ gene & $1.43 \pm 1.62$ & $0.17 \pm 1.75$ & 0.14 \\
\hline
\end{tabular}

Group A- patients without histopatological symptoms of osteitis; Group B- patients with histopatological symptoms of osteitis; CRS- chronic rhinosinusitis, NSAID- nonsteroidal anti-inflammatory drug. findings, asthma and NSAID hypersensitivity (Table 5). In the studied population, we found significant differences between group A and B with respect to IL-6 ( $\mathrm{p}=0.0003)$, IL-11 ( $\mathrm{p}=$ $0.02)$ and TNF- $\alpha$ gene expression in bone tissue $(p=0.0035)$. There was no significant difference between the group with osteitis (group A) and without osteitis (group B) with respect to IL-1 $\beta$ and TGF- $\beta$ genes expression (Table 5).

\section{DISCUSSION}

Osteitis or bone remodeling in CRS usually results in a mixture of neo-osteogenesis and osteolytic lesions, but it is evident that the homeostatic balance between osteoclasts and osteoblasts is disrupted, regardless of the final phenotype. The pressure effects of polyps and the presence of inflammatory mediators such as IL-1, TNF- $\alpha$, and prostaglandin have been proposed as causes of bone remodeling and osteitis ${ }^{(8,9)}$. IL-1, one of the most important proinflammatory cytokines, is a potent transmitter between cells during inflammatory reactions. Practically all cells in the organism respond to an IL-1 stimulus through specific cell membrane receptors. Thus, IL-1 is a non-specific cytokine present in large amounts whenever an important inflammatory process exists ${ }^{(15)}$. IL-1 $\beta$, as well as TNF- $\alpha$, were reported to up-regulate the expression of intercellular adhesion molecule-1 (ICAM-1) and vascular cell adhesion molecule-1 (VCAM-1), which enhances leukocyte infiltration into the nasal mucosa ${ }^{(16)}$.

We have demonstrated that in some patients with CRS and coexisting symptoms of osteitis some inflammatory markers genes expression are increased in this population. The stimulus responsible for this phenomenon remains unclear, but it could be a result of ongoing inflammatory response in the underlying sinus bone. On the basis of these observations osteitis or bone involvement in CRS might be important in prognosis of disease, and the understanding and management of osteitis should be emphasized in the treatment of chronic rhinosinusitis. So far, many hypotheses for CRS pathogenesis have been suggested, but most studies have focused on nasal polyps and diseased mucosa. Chronic inflammatory diseases of the nasal and paranasal sinuses are not limited to the overlying mucosa but also involve the underlying sinus bone. Although the primary lesion of CRS occurs in the nasal mucosa, the bone underneath the mucosa is also affected and interacts with the tissues that surround it. Previous studies have shown radiological and histopathological evidence of bone involvement in $\mathrm{CRS}^{(2-6)}$. It is important to investigate the inflammatory mechanisms that drive chronic rhinosinusitis. Better methods are needed to uncover the pathological process of chronic inflammation to understand the crucial cellular elements, cytokines, mediators and genetic factors involved in this process. Future studies of this subject could consider not only the role of TGF$\beta$, but rather the role of the entire TGF- $\beta$ superfamily, including of bone morphogenetic proteins (BMPs). According to the preliminary results of our study, we propose that the definition 
of rhinosinusitis, as an inflammatory process involving the mucosa of the nose and one or more sinuses, should be expanded deeper to include an inflammatory process in the bony structures underlying the mucosa.

\section{DECLARATION OF INTEREST}

The authors report no conflicts of interest. The authors alone are responsible for the content and writing of the paper.

\section{REFERENCES}

1. Fokkens W, Lund V, Mullol J; European Position Paper on Rhinosinusitis and Nasal Polyps group.European position paper on rhinosinusitis and nasal polyps 2007. Rhinol Suppl. 2007; 20: 1-136.

2. Kennedy DW, Senior BA, Gannon FH, et al. Histology and histomorphometry of ethmoid bone in chronic rhinosinusitis. Laryngoscope 1998; 108: 502-507.

3. Perloff JR, Gannon FH, Bolger WE, et al. Bone involvement in sinusitis: an apparent pathway for the spread of disease. Laryngoscope 2000; 110: 2095-2099.

4. Park JH, Kim YJ, Sung KJ, et al. Correlation between radiologically identified osteitis and prognosis in chronic rhinosinusitis. Korea J Otolaryngol 2005; 48: 998-1003.

5. Jang YJ, Koo TW, Park SG, et al. Osteitis in patients with chronic paranasal sinusitis evaluated by $99 \mathrm{mTc}-\mathrm{MDP}$ bone SPECT. Korea J Otolaryngol 2001; 44: 392-398.

6. Khalid AN, Hunt J, Perloff JR, et al. The role of bone in chronic rhinosinusitis. Laryngoscope 2002; 112: 1951-1957.

7. Kocak M, Smith TL, Smith MM. Bone involvement in chronic rhinosinusitis. Curr Opin Otolaryngol Head Neck Surg 2002; 10: 4952.

8. Shigeru A, Katsuyuki N, Yasuhiro K, et al. Prostaglandin E2 stimulates osteoclast formation via endogenous IL-1 $\beta$ expressed through protein kinase A. J Immunol 1996; 156: 1931-1936.

9. Nukaga J, Kobayashi M, Shinki T, et al. Regulatory effects of interleukin-1 and prostaglandin E2 on expression of receptor activator of nuclear factor- $\mathrm{\kappa B}$ ligand in human periodontal ligament cells. J Periodontol 2004; 75: 249-259.
10. Takahashi N, Udagawa N, Suda T. A new member of tumor necrosis factor ligand family, ODF/OPGL/TRANCE/RANKL, regulates osteoclast differentiation and function. Biochem Biophys Res Commun 1999; 256: 449-455.

11. Suda T, Takahashi N, Udagawa N, et al. Modulation of osteoclast differentiation and function by the new members of the tumor necrosis factor receptor and ligand families. Endocr Rev 1999; 20: 345-357.

12. Kanzaki H, Chiba M, Shimizu Y, et al. Dual regulation of osteoclast differentiation by periodontal ligament cells through RANKL stimulation and OPG inhibition. J Dent Res 2001; 80: 887-891.

13. Wong BR, Josien R, Lee SY, et al. The TRAF family of signal transducers mediates NF-activation by the TRANCE receptor. J Biol Chem 1998; 273: 28355-28359.

14. Wang X, Moylan B, Leopold DA, et al. Mutation in the gene responsible for cystic fibrosis and predisposition to chronic rhinosinusitis in the general population. JAMA. 2000; 284: 1814-1819.

15. Yang-Gi M, Kang Soo L. The role of cytokines in rhinosinusitis. J Korean Med Sci 2000; 15: 255-259.

16. Yamamoto Y, Ikeda K, Watanabe M, et al. Expression of adhesion molecules in cultured human nasal mucosal microvascular endothelial cells activated by interleukin- 1 beta or tumor necrosis factor-alpha: effects of dexamethasone. Int Arch Allergy Immunol. 1998; 117: 68-77.

\section{Marek Postuła, MD}

Department of Experimental and Clinical Pharmacology

Medical University of Warsaw

ul. Krakowskie Przedmieście 26/28 00-927 Warsaw

Poland

Tel: +48-502-138-257

Fax: +48-228-262-116

E-mail: marekpostula@wam.edu.pl 